\title{
MEMS PIEZOELECTRIC ENERGY HARVESTER POWERED WIRELESS SENSOR MODULE DRIVEN BY NOISY BASE EXCITATION
}

\author{
Sijun Du ${ }^{1,2}$, Yu Jia ${ }^{3}$,Emmanuelle Arroyo ${ }^{1}$, Sandra Fernandez ${ }^{4}$, Stephen T. Riches ${ }^{5}$, and Ashwin A. \\ Seshial \\ ${ }^{1}$ Department of Engineering, University of Cambridge, Cambridge, UK, \\ ${ }^{2}$ Department of Electrical Engineering and Computer Sciences, University of California at Berkeley, \\ Berkeley, CA, USA, \\ ${ }^{3}$ School of Engineering and Applied Science, Aston University, Birmingham, UK, \\ ${ }^{4}$ McLaren Applied Technologies, Woking, UK and \\ ${ }^{5}$ Tribus-D, Cambridge, UK
}

\begin{abstract}
Despite recent advances in MEMS vibration energy harvesting and ultra-low power wireless sensors, designing a wireless sensor system entirely powered by a single MEMS device under noisy base excitation has remained a challenge. This paper presents a wireless sensor system co-integrated with a single MEMS piezoelectric vibration energy harvester chip excited by band-limited large amplitude noisy vibration characteristic of an automotive application. The use of soft stoppers in the MEMS package enables the harvesters to operate at an excitation level of $10 \mathrm{~g}(\mathrm{rms})$. A custom thick AlN (Aluminum Nitride) piezoelectric process is employed to fabricate the MEMS harvesters with a single MEMS chip generating $179 \mu \mathrm{W}$ rectified power under these excitation conditions. A low-power wireless sensor module and a receiver module were also designed and demonstrated in this work. Experiments show that the wireless sensor module can be powered solely by the MEMS energy harvester commencing from the cold state. Successful wireless data transmission and receival of sensor data packets are recorded under representative conditions.
\end{abstract}

\section{KEYWORDS}

Energy harvesting, piezoelectric transducers, MEMS, soft stoppers, rectification, power management, wireless sensor networks.

\section{INTRODUCTION}

MEMS inertial vibration energy harvesters have drawn much research interest in the past decade due to the potential for enabling self-powered sensors for automotive and other harsh environment applications where batteries are not feasible power sources, or the costs of battery replacement are prohibitive. However, most MEMS inertial energy harvesters have demonstrated relatively high output power only under steady sinusoidal excitation matched to the resonance of the device, and reliability under high vibration levels remains an unaddressed challenge. Besides, due to the relatively high power consumption of wireless transceivers and certain sensors, the development of a fully self-powered wireless sensor system also requires significant co-optimization of the associated circuits, communication protocols, power management approaches and developing suitable energy-

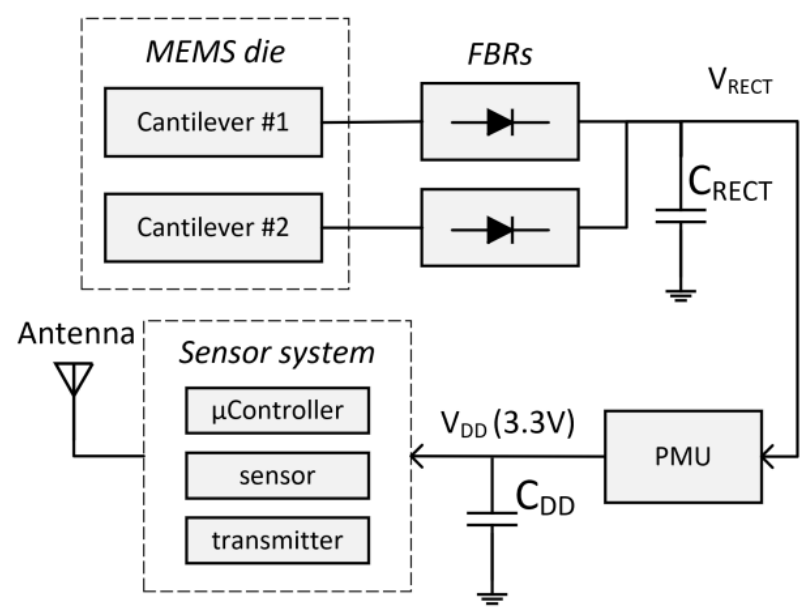

Figure 1: Block diagram schematic of the self-powered wireless sensor module.

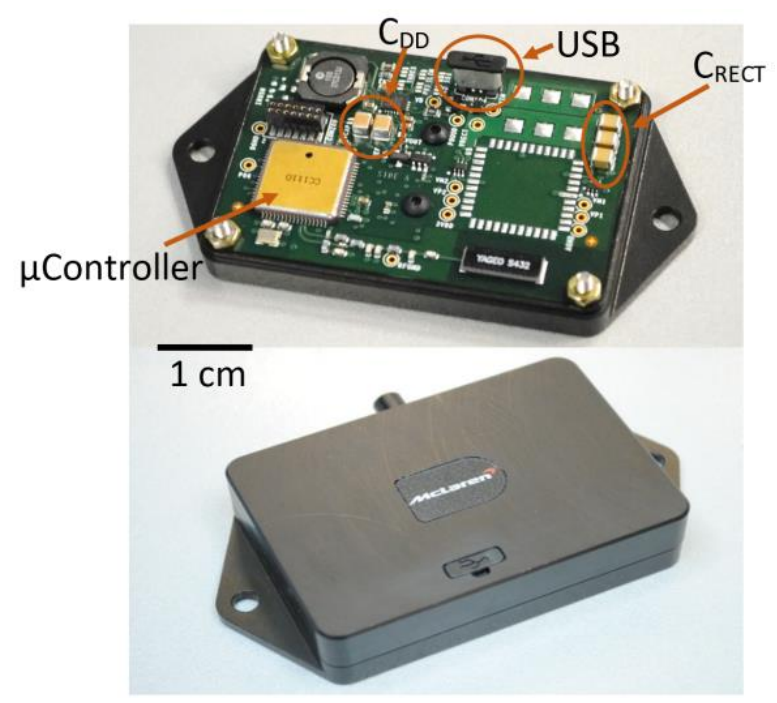

Figure 2: System board with a MEMS piezoelectric energy harvester, a microcontroller, a power management unit (PMU), sensor and wireless transmitter.

neutral rectification interfaces for MEMS vibration energy harvesters. Of the various transduction principles, MEMS piezoelectric vibration energy harvesters (PVEHs) have received significant interest due to the relative scalability of the transduction approach and thin-film processing compatibility of piezoelectric materials such as aluminum nitride $(\mathrm{AlN})$ and zinc oxide $(\mathrm{ZnO})$. 
In previous work, Elfrink et al. [1] demonstrated a MEMS harvester powered wireless sensor node with the harvester generating $10 \mu \mathrm{W}$ DC power under steady sinusoidal conditions. This work was further progressed by the same group towards the development of a harvester-powered wireless sensor under noisy conditions generating $28.9 \mu \mathrm{W}$ ac power across a matched load for a TPMS application [2]. However, though subsequent work demonstrated MEMS harvesters delivering sufficient DC power (10's $\mu \mathrm{W})$ for automotive applications, concerns around reliability under high shock and vibration have remained [3]. In other related work, Fan et al. [4] and
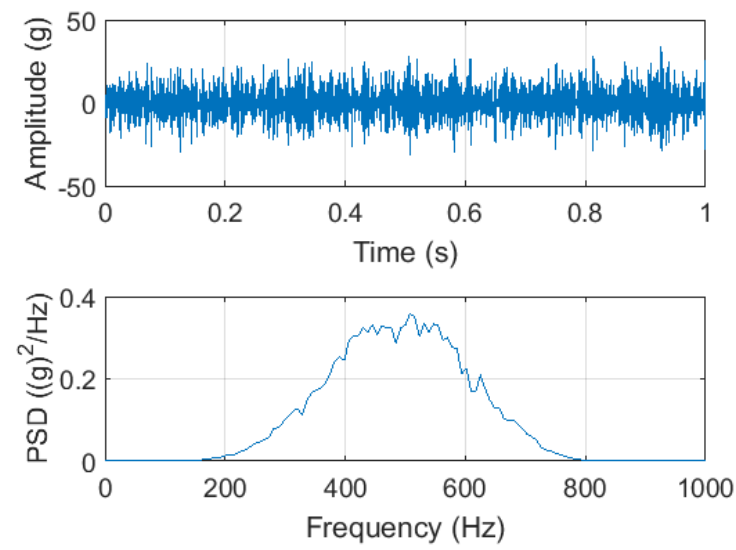

Figure 3: Representative time domain and PSD plots for the base vibration.

Tang et al. [5] previously demonstrated wireless temperature sensor systems powered by miniaturized PVEHs; however, the PVEHs are excited at their natural frequency and at low excitation levels. Aktakka et al. [6] demonstrated a PZT-on-silicon micromachining process incorporating a tungsten proof-mass enabling inertial MEMS energy harvesters to generate $95 \mu \mathrm{W}$ raw output power [7] under $1 \mathrm{~g}$ sinusoidal excitation at the natural frequency. Recent work by our group [8] has demonstrated MEMS energy harvesters generating up to $186 \mu \mathrm{W}$ rectified power under sinusoidal excitation conditions for AIN MEMS energy harvesters cointegrated with CMOS rectification circuits.

In this paper, we present results from a self-powered wireless sensor system co-integrated with a MEMS energy harvester, realizing cold startup and data transmission.

\section{SYSTEM DESCRIPTION}

A typical self-powered wireless sensor system consists of an energy harvesting device, rectification and power management circuitry and a wireless sensor module. Figure 1 shows the block diagram of the system presented in this work. In the context of vibration energy harvesting, the device is usually designed to accommodate the target frequency characteristics and to output a high output power under specific environmental conditions. Rectification of the harvester output is required before further processing. Although active rectifiers, such as bias-flip rectifiers [8], have high energy efficiency for low input excitation, passive full-bridge rectifiers are employed in this work for simplicity since the input excitation is high. The sensor module consists of a micro-controller, temperature and pressure sensors, a wireless transmitter, I/O circuits, signal modulation circuits, ADC, and an antenna. A photo of the module is shown in Figure 2. The system includes one MEMS device consisting of two cantilevers, two full-bridge rectifiers (FBRs) for power rectification, a power management unit (PMU) to provide DC supply and the

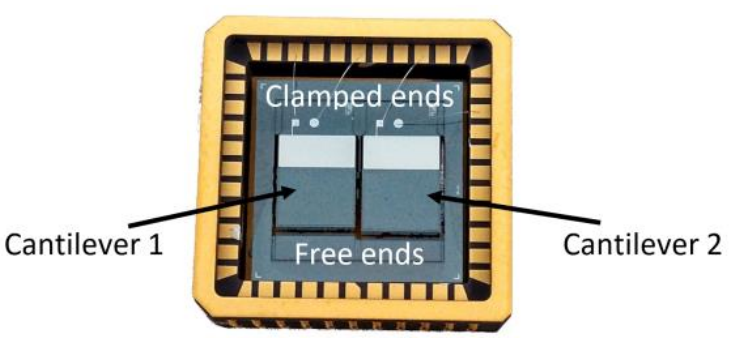

Figure 4: Photo of a MEMS piezoelectric energy harvester in the thick AlN process comprising of two packaged MEMS cantilevers

sensor system consisting of a micro controller, a sensor and a wireless transmitter.

\section{Fabrication process}

In order to power the entire wireless sensor system, a single MEMS device ( $1 \mathrm{~cm} \times 1 \mathrm{~cm}$ chip area) is employed. The device is fabricated in a custom MEMS process incorporating a $3 \mu \mathrm{m}$ thick AlN (Aluminum Nitride) piezoelectric film [8]. The thick AlN and doped silicon layers result in an increased natural frequency of the cantilevered harvesters with natural frequencies situated in the vibration band of interest $(400-600 \mathrm{~Hz})$.

Table 1: Design parameters and measured results of the two cantilevers in a MEMS chip.

\begin{tabular}{|c|c|c|}
\hline & Cantilever 1 & Cantilever 2 \\
\hline $\mathrm{L}$ & $5100 \mu \mathrm{m}$ & $5300 \mu \mathrm{m}$ \\
\hline $\mathrm{W}$ & $4200 \mu \mathrm{m}$ & $4200 \mu \mathrm{m}$ \\
\hline $\mathbf{f}$ & $511 \mathrm{~Hz}$ & $468 \mathrm{~Hz}$ \\
\hline P $_{\text {RECT }}$ & $93 \mu \mathrm{W}$ & $86 \mu \mathrm{W}$ \\
\hline
\end{tabular}

\section{MEMS design}

The entire system is designed to operate under large amplitude noisy base excitation levels corresponding to an automotive application. The representative time series and frequency response characteristics corresponding to the base excitation in this scenario are illustrated in Figure 3. The frequency is centered at around $475 \mathrm{~Hz}$ spanning a bandwidth of around $200 \mathrm{~Hz}$ at an excitation level of 10 $\mathrm{g}(\mathrm{rms})$. This represents a vibration profile that matches the characteristics of measured vibration at a monitoring location of interest on a Formula One car. To accommodate the wide frequency range, two cantilevers with slightly different dimensions are designed in a single MEMS die. Figure 4 shows the photo of the MEMS devices following their insertion into a ceramic chip carrier and wirebonding. Table 1 provides a summary of 
the design parameters and measured natural frequency and rectified power output for the two cantilevers when subject to the noisy base excitation profile.

\section{Packaging}

The excitation level is as high as $10 \mathrm{~g}(\mathrm{rms})$ and it is essential to ensure that the packaged MEMS device does

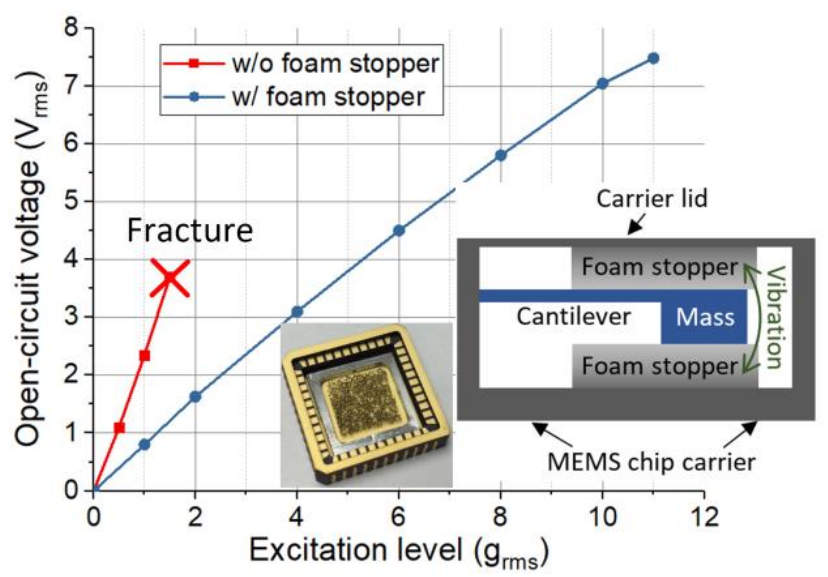

Figure 5: Comparison of measured excitation tolerance for MEMS devices with and without soft stoppers.

not fail under this excitation level. To avoid failure under an excitation of $10 \mathrm{~g}(\mathrm{rms})$, soft foam stoppers were inserted above and below both cantilevers. Figure 5 shows the measured open-circuit voltage (rms) of MEMS devices with and without soft stoppers. The stoppers are inserted such that the cantilever is sandwiched between the top and bottom stoppers, providing increased tolerance to high base excitation levels. The results show that when there is no stopper, cantilevers fracture at around $1.5 \mathrm{~g}(\mathrm{rms})$ corresponding to a peak open-circuit voltage of $3.6 \mathrm{~V}$. Following insertion of soft stoppers, the cantilevers keep operating at excitation levels up to 11 $\mathrm{g}(\mathrm{rms})$ and the open-circuit voltage peaks at $7.5 \mathrm{~V}$. The

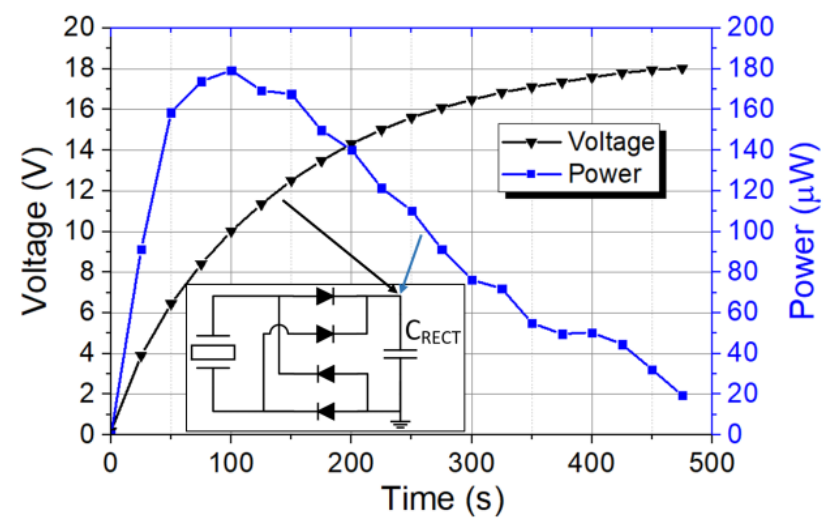

Figure 6: Measured rectified voltage and rectified power at the capacitor $C_{R E C T}$ at $10 \mathrm{~g}(\mathrm{rms})$ excitation for one MEMS die (two cantilevers).

soft stoppers, thus, allow the device to operate at much higher excitation levels enabling higher output power.

\section{EXPERIMENTAL RESULTS}

The rectified voltage and power are first measured for

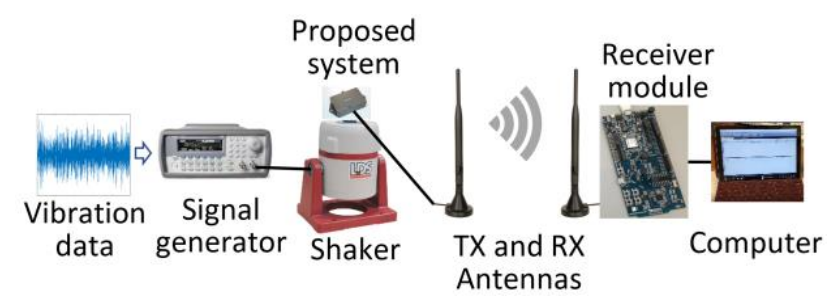

Figure 7: Experimental setup to test the proposed fully self-powered wireless sensor system.

the MEMS vibration energy harvester chip (comprising the two cantilevers) under $10 \mathrm{~g}(\mathrm{rms})$ excitation. Figure 6 summarizes the measured results. The voltage labeled in the figure is the voltage measured across the capacitor $\mathrm{C}_{\mathrm{RECT}}$, shown in Figure 1. In order to rectify the power generated by two cantilevers, two full-bridge rectifiers are employed and connected to a single capacitor $\mathrm{C}_{\mathrm{RECT}}$. The results show that, after 500 seconds of continuous base excitation at $10 \mathrm{~g}(\mathrm{rms}), V_{R E C T}$ can be charged to around 18 $\mathrm{V}$ if the PMU is not present. The measured $V_{R E C T}$ voltage is noted every 25 seconds. The power curve in Figure 7 is calculated from the voltage curve with the following equation:

$$
P(\mathrm{t})=0.5 \mathrm{C}_{\text {RECT }}\left(V_{R E C T}(\mathrm{t})^{2}-V_{R E C T}(\mathrm{t}-1)^{2}\right)
$$

where $V_{R E C T}(\mathrm{t})$ is the $V_{R E C T}$ voltage at time $\mathrm{t}$, and $V_{R E C T}(\mathrm{t}-$ $1)$ is the $V_{R E C T}$ voltage value measured at the time instant $25 \mathrm{~s}$ prior. It can be seen that the peak rectified power attains $179 \mu \mathrm{W}$, which is sufficient to power the wireless sensor system.

\section{Measurement setup}

Figure 7 shows the experimental setup to demonstrate the vibration-powered wireless sensor system. The vibration data is first programmed into a signal generator. Prior to commencing the experiments, an accelerometer is fixed on the shaker to calibrate the base excitation and adjust the applied excitation level to $10 \mathrm{~g}(\mathrm{rms})$. After this calibration step is complete, the system is mounted on the shaker. Before turning on the signal generator, the remaining charge in capacitors $\mathrm{C}_{\mathrm{RECT}}$ and $\mathrm{C}_{\mathrm{DD}}$ in the system (can be found in Figure 2) is manually cleared using a wire to short the two electrodes of each capacitor. This is to ensure that the system is at the cold state before it is charged from the MEMS energy harvesters. A TX antenna is connected to the wireless sensor module for data transmission. A RX antenna is placed a few meters away from the TX antenna and it is connected to a receiver module. The receiver module is connected to a computer via a USB cable to record the received data packets.

\section{Measured results}

Figure 8 shows the measured waveforms of acceleration level, $V_{R E C T}$ and $V_{D D}$. The figure shows that $V_{R E C T}$ and $V_{D D}$ are near $0 \mathrm{~V}$ at the beginning. This means that the system is fully discharged before excitation is present. About 25 seconds later, the signal generator is turned on and the system is subject to the $10 \mathrm{~g}(\mathrm{rms})$ 
excitation profile as shown in the measured accelerometer waveforms. The full-bridge rectifiers first start operating to charge the capacitor $\mathrm{C}_{\mathrm{RECT}}$ (labeled in Figure 1). After $V_{R E C T}$ attains a pre-configured threshold, the PMU starts transferring energy from $\mathrm{C}_{\mathrm{RECT}}$ to $\mathrm{C}_{\mathrm{DD}}$ to build up the $\mathrm{DC}$ supply for the sensor system, $V_{D D}$. As $V_{D D}$ gradually increases, $V_{R E C T}$ is nearly constant as all the harvested energy is transferred to $\mathrm{C}_{\mathrm{DD}}$. After $V_{D D}$ attains the preset 3.3 $\mathrm{V}$ value, the sensor system starts operating to collect and wirelessly transmit sensor data. During this period, $V_{R E C T}$ rises higher, providing sufficient energy for the next data transmission. The highest $V_{R E C T}$ value is set at $5.5 \mathrm{~V}$. The data transmission is configured to occur every 1 minute. It can be clearly seen from the figure that $V_{R E C T}$ is slightly discharged every $1 \mathrm{~min}$ while $V_{D D}$ is maintained at $3.3 \mathrm{~V}$. The consumed energy in $\mathrm{C}_{\mathrm{RECT}}$ is used for wireless data transmission. It can be found that the first data transmission consumes more energy than subsequent transmissions due to TX circuitry initializations. The transmission can be configured to occur more frequently since $V_{R E C T}$ can be charged in a short time after each transmission.

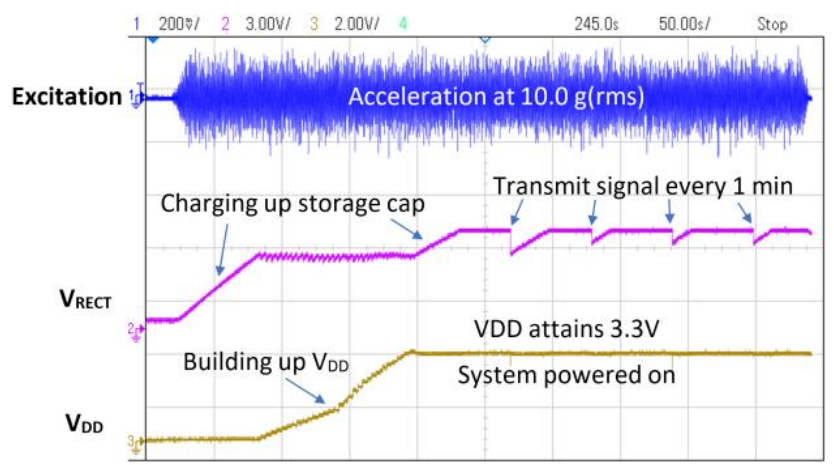

Figure 8: Measured waveforms (500 s) while the entire wireless sensor system is solely powered by the MEMS energy harvester from the cold state.

\section{SUMMARY}

In this paper, a vibration-powered wireless sensor system is demonstrated. A custom MEMS process is employed to fabricate thick piezoelectric layer devices to deliver high output power under noisy base excitation conditions. Soft stoppers are employed within the MEMS package to limit motion under high amplitude base excitation, allowing the energy harvesters operating at excitation levels as high as $10 \mathrm{~g}(\mathrm{rms})$ while delivering high output power. A sensor system module comprising sensors, micro-processor, power management and peripheral circuits is designed to operate within the power budget for a single MEMS die. Experimental results show that the rectified power harvested by one single MEMS device peaks at $179 \mu \mathrm{W}$ using passive full-bridge rectifiers under $10 \mathrm{~g}(\mathrm{rms})$ noisy base excitation. Successful continuous wireless transmission of sensor data is demonstrated under these conditions. Future work required to enable application at scale will need to consider manufacturability (including integration of soft stoppers), limits to robustness, integration with CMOS rectification interfaces, power management and energy storage, and further design co-optimization and miniaturization of the wireless sensor module.

\section{ACKNOWLEDGEMENTS}

This work was supported by Innovate UK (Grant number 102152) and the UK Engineering and Physical Sciences Research Council (EP/N021614/1).

\section{REFERENCES}

[1] R. Elfrink et al., "First autonomous wireless sensor node powered by a vacuum-packaged piezoelectric MEMS energy harvester," 2009 IEEE International Electron Devices Meeting, Baltimore, MD, USA, 2009, pp. 543-546.

[2] Z. Wang et al., "Large power amplification of a piezoelectric vibration energy harvester excited by random vibrations," 2013 IEEE International Conference on MicroElectroMechanical Systems (IEEE MEMS), Taipei, Taiwan, 2013, pp. 106-109.

[3] M. Renaud et al., Improved mechanical reliability of MEMS piezoelectric vibration energy harvesters for automotive applications, 2014 IEEE International Conference on MicroElectroMechanical Systems (IEEE MEMS), 2014, San Francisco, CA, USA, pp. 568-571.

[4] S. Fan et al., "mm-Scale and MEMS piezoelectric energy harvesters powering on-chip CMOS temperature sensing for IoT applications," 2017 19th International Conference on Solid-State Sensors, Actuators and Microsystems (TRANSDUCERS), Kaohsiung, 2017, pp. 1848-1850.

[5] Q. C. Tang, Q. S. He, M. Y. Li, C. Dong, D. C. Xu and $\mathrm{X}$. Li, "A self-powered wireless sensing node for event-driven alerting based on a bi-stable vibration energy harvester," 18th International Conference on Solid-State Sensors, Actuators and Microsystems (TRANSDUCERS), Anchorage, AK, 2015, pp. 12151218.

[6] E. Aktakka, R. Petersen and K. Najafi, "Thinned-PZT on SOI process and design optimization for piezoelectric inertial energy harvesting, $201116^{\text {th }}$ International Conference on Solid-State Sensors, Actuators and Microsystems (TRANSDUCERS), Beijing, China, 2011, pp. 1649-1652.

[7] E. Aktakka and K. Najafi, "A micro inertial energy harvesting platform with self-supplied power management circuit for wireless sensor nodes, IEEE Journal of Solid-State Circuits, vol. 49, no. 9, pp. 2017-2029, 2014.

[8] S. Du, Y. Jia, C. Zhao, G. A. J. Amaratunga, and A. A. Seshia, "A Fully Integrated Split-Electrode SSHC Rectifier for Piezoelectric Energy Harvesting," IEEE Journal of Solid-State Circuits, 2019, in press. (DOI: 10.1109/JSSC.2019.2893525).

\section{CONTACT}

*A. Seshia, tel: +44 1223 760333; aas41@ @am.ac.uk 